\title{
On the feasibility of using an extracted polarized antiproton beam of HESR with a solid polarized target
}

\section{G.M. Gurevich ${ }^{a}$, A.A. Lukhanin ${ }^{b}$, F. Maas ${ }^{c}$, Yu.A. Plis ${ }^{* d}$, A.O. Sidorin ${ }^{d}$,}

\section{A.V. Smirnov ${ }^{* d}$, A. Thomas ${ }^{e}$ and Yu.A. Usov ${ }^{d}$}

${ }^{a}$ Institute for Nuclear Research, RAS,

117312 Moscow, Russia

${ }^{b}$ Kharkiv Institute of Physics and Technology,

Kharkiv, Ukraine

${ }^{c}$ Helmholtz-Institut Mainz, Universität Mainz,

D-55099, Germany

${ }^{d}$ Joint Institute for Nuclear Research,

141980 Dubna, Russia

e Institut für Kernphysik, Universität Mainz,

D-55099, Germany

E-mail: vury1939dmail.ru, smirnovdjinr.ru

It seems realizable to accelerate and store polarized antiprotons in the high-energy storage ring (HESR) of the future GSI Facility for Antiproton and Ion Research (FAIR). Here we accept that all the problems connected with antiproton production and depolarization are solved.

At HESR the planned internal coasting beam will reach $10^{11}$ antiprotons in the momentum range 1.5-15 GeV/c. One might try to extract halo antiprotons, which cannot be utilised at the PANDA facility, by means of a silicon crystal and magnetic septum, for experiments at a solid polarized target.

The beam lifetime for HESR is $\approx 1$ hour, while for the Fermilab it equaled $\approx 70$ hours. It can be expected, that in HESR the substantial share of antiprotons lost due to interaction with pellets comes to the halo.

The simulation has been done with the BETACOOL code for the PANDA parameters with using the barrier bucket and electron cooling systems. The longitudinal acceptance was chosen equal to the effective barrier bucket height. It means that particle will be lost after interaction with the target pellet if the energy decreasing is larger than the barrier height.

For the cycle duration equal to 1 hour an average intensity will be $\sim 10^{6}$ antiprotons/s, which, for the typical $20 \mathrm{~cm}$ long polarized target, corresponds to luminosity $\sim 10^{30} \mathrm{~cm}^{-2} \mathrm{~s}^{-1}$.

XVIth International Workshop in Polarized Sources, Targets, and Polarimetry, PSTP2015,

14-18 September 2015,

Bochum, Germany

\footnotetext{
* Speaker.
} 


\section{Introduction}

It seems realizable to accelerate and store polarized antiprotons in the high-energy storage ring (HESR) of the future GSI Facility for Antiproton and Ion Research (FAIR). Here we assume that all the problems connected with antiproton production and depolarization are solved [四]. Here we consider the possibility to use the solid polarized proton target with an extracted polarized antiproton beam for physics experiments.

It is well known, that high-energy protons could be efficiently extracted from an accelerator using the technique of channeling in a bent crystal. As it was shown recently, this technique also permits to extract the particles from the beam halo in a proton collider without interfering in the main experiment [2]. The principle of a parasitic extraction of protons with a bent crystal is very simple: placing a bent crystal on a goniometer in the halo of the circulating proton beam. Align the crystalline planes with the momentum of the protons in the halo, in order to allow channeling (and extraction). The main beam and thus the collider experiments would be undisturbed, and the protons used for extraction are only the ones which otherwise would be lost.

Experiments on the Tevatron (FNAL) at the energy $900 \mathrm{GeV}$ confirmed computer simulations and provided the extraction coefficient about $10^{-6}$, which for $10^{12}$ circulating protons resulted in the extracted beam of $10^{6}$ protons/s [B]].

Similar results were received at the CERN SPS. With a $120 \mathrm{GeV}$ coasting beam of about $5 \times 10^{11}$ protons typically $6 \times 10^{5}$ protons per second were extracted [䧃].

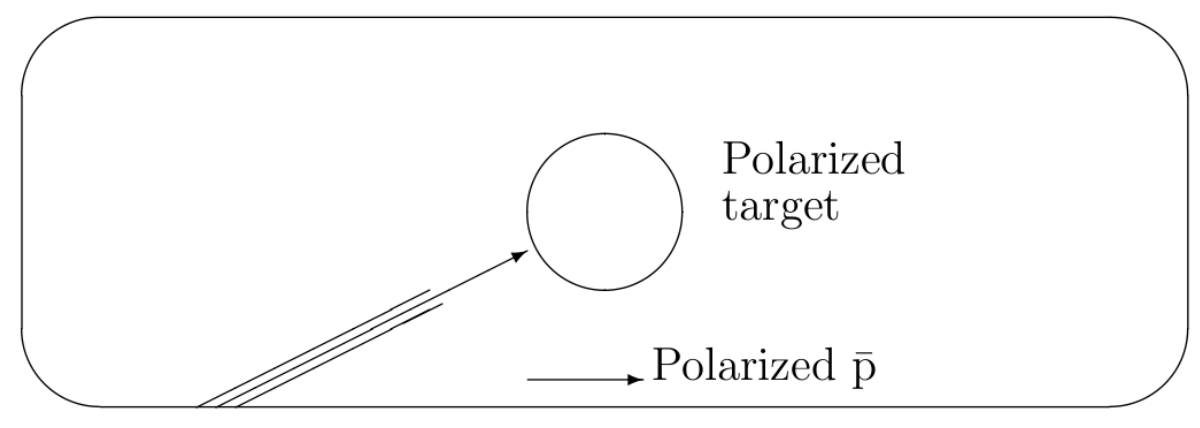

Figure 1: Scheme of the polarized antiproton extraction

It is also known [1], 目], that for heavy negative particles (e.g., antiprotons) the method of the beam deflection by a bent crystal in the mode of standard planar channeling is inefficient due to the fast dechanneling. Instead, stochastic mechanism the multiple scattering of negative particles on the bent chains of crystal atoms could be helpful [6]. Experiments at CERN confirmed the computer simulation results for the stochastic mechanism for the extracted negative pion beam deflection by bent crystals [四].

Authors [ [ $]$ point out that this method could be used for the antiproton beam extraction from an accelerator. The simulation [U] has been carried out for the beam of 2000 antiprotons with the energy $10 \mathrm{GeV}$, incident on the silicon crystal in the direction of $<110>$ axis for $\psi_{c}=145 \mu \mathrm{rad}$. Beam divergence was taken equal to $\approx 10 \mu \mathrm{rad}$, bending radius $R=1 \mathrm{~m}$, crystal thickness $L=1$ $\mathrm{mm}$, bending angle $\alpha=L / R=1 \mathrm{mrad}$. So, it seems that this method is not effective. 
In HESR [ [8] the planned internal circulating beam could reach $10^{11}$ antiprotons in the momentum range $1.5-15 \mathrm{GeV} / \mathrm{c}$ (energies $0.83-14.1 \mathrm{GeV}$ ). The beam lifetime in the working mode with the use of hydrogen pellets changes from 0.5 hour at a minimum energy to 2 hours at a maximum energy [ $[$ ]. Furthermore, the mechanism of the halo formation in HESR is different in comparison with the case of the FNAL Tevatron where the beam lifetime reaches 70 hours. This implies, that the extraction coefficient for HESR could turn out essentially higher than for the Tevatron.

Proceeding from the mentioned above, we considered two possibilities for obtaining an EXTRACTED antiproton beam in HESR with the purpose to use it independently for the study of polarization effects. Polarized antiprotons would open new possibilities in hadron physics providing access to a wealth of single and double spin observables in proton-antiproton interactions [ए0]].

\section{Extraction with a bent crystal}

It is suggested to use the stochastic mechanism for the antiproton deflection by a bent silicon crystal. The simulation [ $\mathbb{[}$ ] has been carried out for the beam of 2000 antiprotons with the energy $10 \mathrm{GeV}$, incident on the silicon crystal in the direction of $<110>$ axis for $\psi_{c}=145 \mu \mathrm{rad}$. Beam divergence was taken equal to $\approx 10 \mu \mathrm{rad}$, bending radius $R=1 \mathrm{~m}$, crystal thickness $L=1 \mathrm{~mm}$, bending angle $\alpha=L / R=1 \mathrm{mrad}$. In this case the maximum beam deflection can reach 0.25 mrad and the distribution width 2 mrad. At HESR one might try to extract halo antiprotons, which cannot be utilised by the PANDA facility, with the assistance of magnetic septum (Fig. W). The

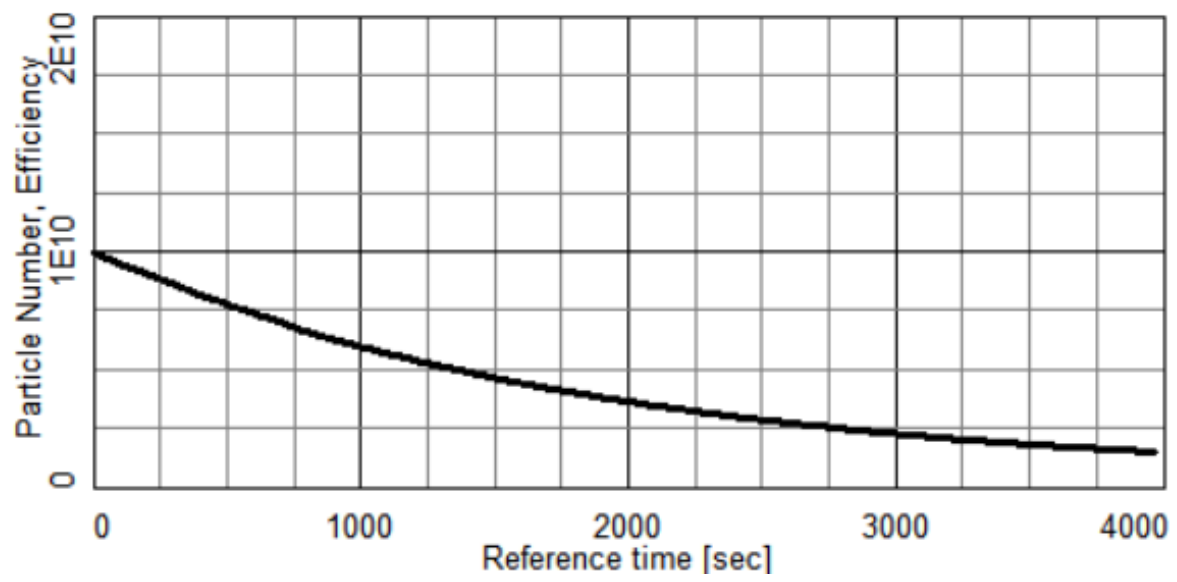

Figure 2: Particle losses due to the longitudinal acceptance and atomic reaction in the internal target.

initial direction of polarization is vertical, it can be rotated in any direction before reaching the polarized target.

It should be noted that the halo formation mechanism in HESR differs from that in the FNAL storage ring, where the in-beam scattering is the main process. In HESR at the minimal momentum the main contribution comes from Coulomb scattering, while at the maximal momentum - from nuclear processes [ $[$ ]. The beam lifetime for HESR is about 1 hour, while for FNAL is about 70 hours. It can be expected, that in HESR the substantial share of antiprotons lost due to interactions with pellets comes to the halo. However, the computer simulation is necessary. 
Simulation was done with BETACOOL code [[13] for PANDA parameters with using the barrier bucket and electron cooling systems [144]. Main simulation parameters are presented in Table ஐ. The longitudinal acceptance was chosen equal to the effective barrier bucket height. It means that the particle will be lost after interaction with the target pellet if the energy decreasing is larger than the barrier height.

If the particle losses are defined by the longitudinal acceptance and by the atomic reaction in the internal target (cross section is 0.055 barn) then the loss rate is about $4 \times 10^{6}$ particles per second (Figs. [ [ ] [ ] ). In the case when the particle losses are defined by the longitudinal acceptance only the particle loss rate can be estimated on the level about $1.2 \times 10^{6}$ particles per second (Fig. (4). This value can be used as a simple estimation of the intensity of the slow extracted beam for the choosen parameters. Note that in HESR horizontal dispersion function has mainly positive values. It means that the particles can be extracted to the inner side of the storage ring.

For the estimation of the real intensity of the slow extracted beam the BETACOOL program can be modified in accordance with necessary physical and geometrical models (beam and target parameters, extraction kicker parameters, etc.). The particle loss rates (extracted beam intensity) can be controlled with the different parameters of the barrier bucket and electron cooling systems which also define the quality of the slow extracted beam.

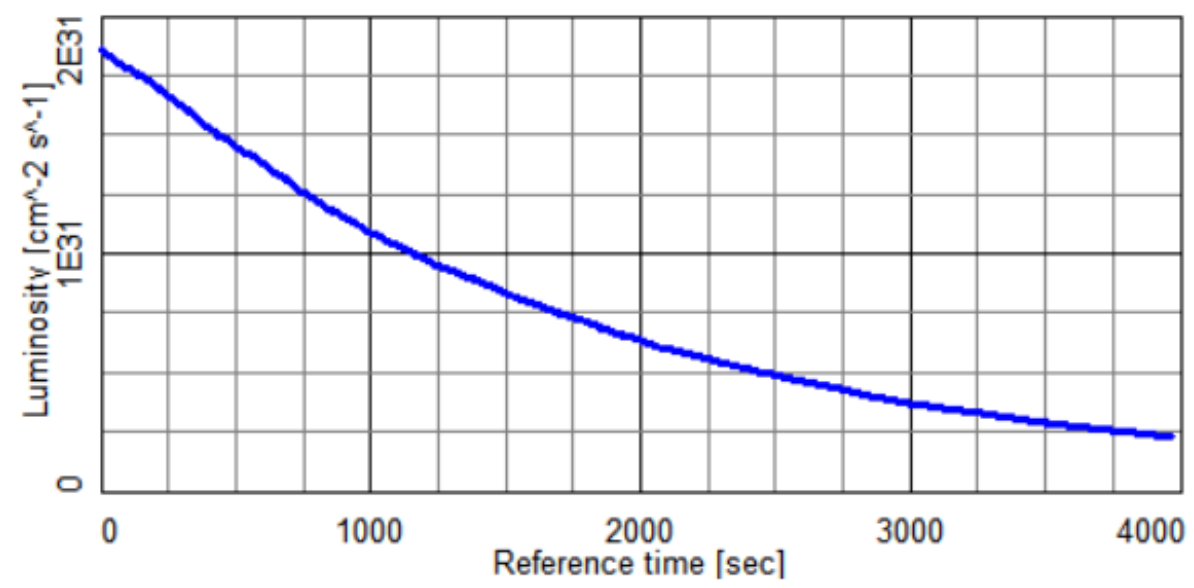

Figure 3: Luminosity evolution due to the longitudinal acceptance and atomic reaction in the internal target.

For a typical $20 \mathrm{~cm}$ long polarized target the luminosity will be about $10^{29} \mathrm{~cm}^{-2} \mathrm{~s}^{-1}$.

\section{Slow resonant extraction}

Since the accumulated antiprotons in HESR are not utilised entirely, it is feasible to extract them slowly at the end of the cycle and send to the polarized target. For example, at slow variation of the radial betatron oscillation frequency the particles get to the instability region and end up in the septum due to the resonance build-up of oscillation amplitude. If the data acquisition stops when the number of particles drops by an order of value, i.e. down to $10^{10}$ or $10^{9}$ (depending on the chosen regime) and the particles are extracted for $10 \mathrm{~s}$ with the efficiency of $10 \%$, then for the cycle duration equal to 1 hour an average intensity will be $3 \times 10^{6}-3 \times 10^{5}$ antiproton/s, which, for the $20 \mathrm{~cm}$ long polarized target, corresponds to the luminosity $3 \times 10^{30}-3 \times 10^{29} \mathrm{~cm}^{-2} \mathrm{~s}^{-1}$. 


\begin{tabular}{|c|c|}
\hline & \\
Antiproton beam, storage ring & \\
\hline Energy, GeV & 8 \\
Particle number & $10^{10}$ \\
Initial emittance, $\pi$ mm mrad & 0.039 \\
Initial momentum spread, dp/p & $1.3 \times 10^{-4}$ \\
Longitudinal acceptance, dp/p & $5 \times 10^{-4}$ \\
\hline Barrier bucket system & \\
\hline Amplitude, $\mathrm{kV}$ & 0.4 \\
Barrier width, \% of circumference & 10 \\
Barrier gap, \% of circumference & 70 \\
Effective barrier height, dp/p & $5 \times 10^{-4}$ \\
\hline Electron cooling system & \\
\hline Electron cooler length, m & 20 \\
Electron beam radius, cm & 0.5 \\
Electron current, A & 0.2 \\
Electron temperature, eV (tran/long) & $1.0 / 5 \times 10^{-4}$ \\
\hline Hydrogen internal target & \\
\hline Effective density, cm ${ }^{-2}$ & $3.67 \times 10^{15}$ \\
Pellet size, mm & 0.028 \\
Beta functions, $\mathrm{m}$ (hor/ver) & $8 / 8$ \\
Cross-section, barn & 0.055 \\
\hline
\end{tabular}

Table 1:

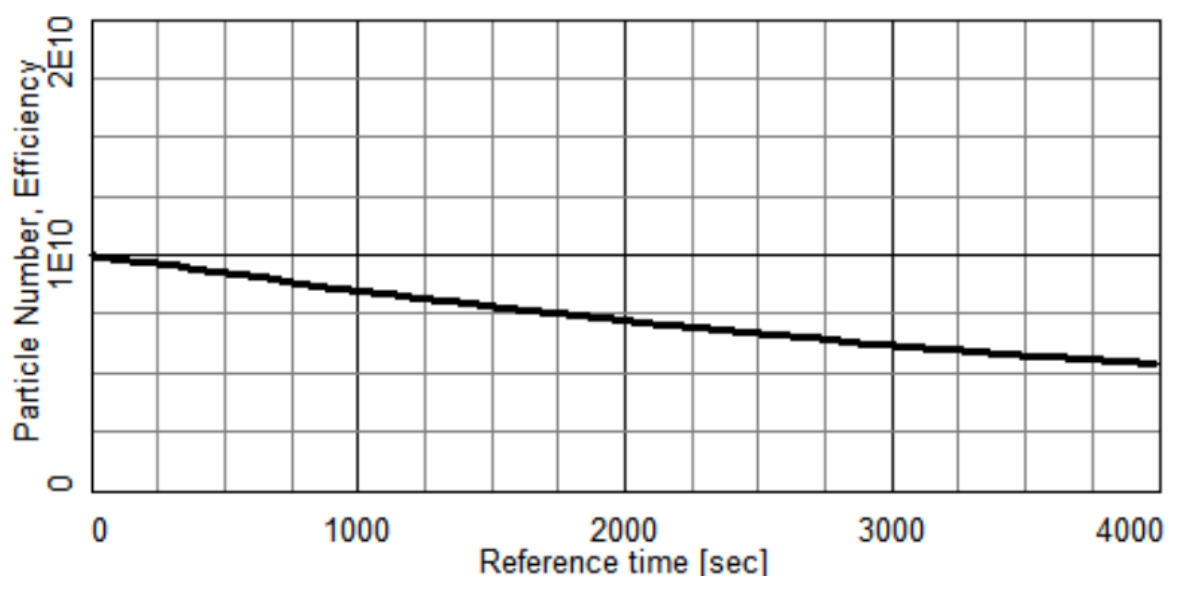

Figure 4: Particle losses due to the longitudinal acceptance. 


\section{Summary}

In summary, an extracted antiproton beam which is to be obtained by the use of one of the described methods could be employed in combination either with a dynamically polarized target (DPT) or with a frozen spin target (FST), depending on parameters of the beam which will be reached as a result of joint efforts of scientists from Dubna and Mainz. Of course, the realization of a similar project is a serious technical problem which could be solved by a close co-operation of all relevant experts solely.

All the results could be applicable for extraction of heavy ions from storage rings (colliders).

\section{References}

[1] A. Lehrach et al., SPIN2004: Proc. of the 16th Int. Spin Physics Symp., Trieste, pp. 742-745.

[2] R. Carrigan et al., Phys. Rev. ST - AC 1 (1998) 022801.

[3] R. Carrigan et al., Phys. Rev.AB 5 (2002) 043501.

[4] K. Elsener et al., Nucl. Instr. \& Meth. B 119 (1996) 215.

[5] V.P. Koshcheev, O.E. Krivosheev., Russian Phys. Journal. 38 (1995) 493.

[6] N.F. Shul'ga, I.V. Kirillin, V.I. Truten', Phys. Lett. B 702 (2011) 100.

[7] W. Scandale et al., Phys. Lett. B 680 (2009) 301.

[8] FAIR Project (subproject HESR) Technical Report. http:// www.gsi.de /fait/reports/btr-e.html.

[9] A. Lehrach et al., Nucl. Instr. \& Meth. A 561 (2006) 289.

[10] P. Lenisa, PSTP 2011: Proc. 14th Int. Workshop on Polarized Sources, Targets and Polarimetry, eds. K. Grigoryev, P. Kravtsov and A. Vasilyev, St. Petersburg, Russia, ISBN 978-5-8673-282-3 (2011) pp. 55-58.

[11] N.F. Shul'ga, I.V. Kirillin, V.I. Truten', http://iap.sumy.org/attacments/files/Kharkov2014/12.pdf.

[12] X. Altuna et al., Phys. Lett. B 357 (1995) 671.

[13] http://betacool.jinr.ru.

[14] A. Smirnov, A. Sidorin, D. Krestnikov. Effective Luminosity Simulation for PANDA Experiment at FAIR. Proceeding of Workshop on Beam Cooling and Related Topics (COOL09). Lanchzhou, China (2009). http://jacow.org. 